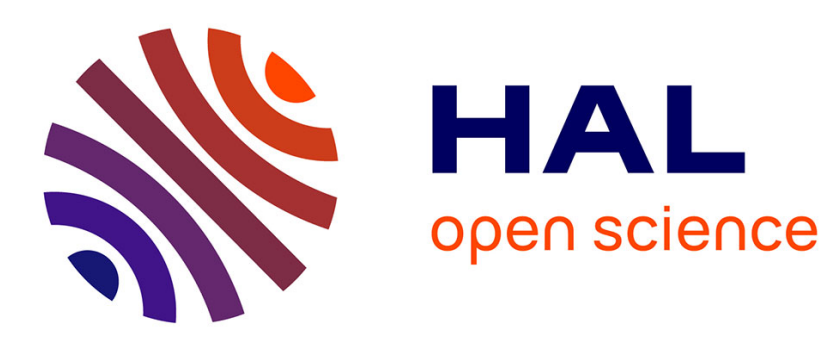

\title{
Homotopy commutative algebra and 2-nilpotent Lie algebra
}

Michel Dubois-Violette, Todor Popov

\section{To cite this version:}

Michel Dubois-Violette, Todor Popov. Homotopy commutative algebra and 2-nilpotent Lie algebra. 2012. hal-00718938

\section{HAL Id: hal-00718938 \\ https://hal.science/hal-00718938}

Preprint submitted on 18 Jul 2012

HAL is a multi-disciplinary open access archive for the deposit and dissemination of scientific research documents, whether they are published or not. The documents may come from teaching and research institutions in France or abroad, or from public or private research centers.
L'archive ouverte pluridisciplinaire HAL, est destinée au dépôt et à la diffusion de documents scientifiques de niveau recherche, publiés ou non, émanant des établissements d'enseignement et de recherche français ou étrangers, des laboratoires publics ou privés. 


\title{
Homotopy commutative algebra and 2-nilpotent Lie algebra
}

Michel Dubois-Violette and Todor Popov

\begin{abstract}
The homotopy transfer theorem due to Tornike Kadeishvili induces the structure of a homotopy commutative algebra, or $C_{\infty}$-algebra, on the cohomology of the free 2-nilpotent Lie algebra. The latter $C_{\infty}$-algebra is shown to be generated in degree one by the binary and the ternary operations.
\end{abstract}

\section{Introduction}

Every Universal Enveloping Algebra (UEA) $U \mathfrak{g}$ of a finite dimensional positively graded Lie algebra $\mathfrak{g}$ belongs to the class of Artin-Schelter regular algebras(see e.g. [4]). As every finitely generated graded connected algebra, $U \mathfrak{g}$ has a free minimal resolution which is canonically built from the data of its Yoneda algebra $\mathscr{E}:=\operatorname{Ext}_{U \mathfrak{g}}(\mathbb{K}, \mathbb{K})$. By construction the Yoneda algebra $\mathscr{E}$ is isomorphic (as algebra) to the cohomology of the Lie algebra (with coefficients in the trivial representation provided by the ground field $\mathbb{K}$ )

$$
\mathscr{E}=\operatorname{Ext}_{U \mathfrak{g}}^{\bullet}(\mathbb{K}, \mathbb{K}) \cong H^{\bullet}(\mathfrak{g}, \mathbb{K})
$$

equipped with wedge product between cohomological classes in $H^{\bullet}(\mathfrak{g}, \mathbb{K})$.

The homotopy transfer theorem of Tornike Kadeishvili [7] implies that the Yoneda algebra $\mathscr{E}=\operatorname{Ext}_{U \mathfrak{g}}^{\bullet}(\mathbb{K}, \mathbb{K})$ has the structure of homotopy associative algebra, or $A_{\infty}$-algebra. Since $\mathscr{E} \cong H^{\bullet}(\mathfrak{g}, \mathbb{K})$ is the cohomology of the exteriour algebra

Michel Dubois-Violette

Laboratoire de Physique Théorique, UMR 8627, Université Paris XI, Bâtiment 210, F-91 405 Orsay Cedex, France e-mail: Michel.Dubois-Violette@ th.u-psud.fr

Todor Popov

Institute for Nuclear Research and Nuclear Energy, Bulgarian Academy of Sciences, 72 Tsarigradsko chaussée, 1784 Sofia, Bulgaria e-mail: tpopov@inrne.bas.bg 
$\wedge \mathfrak{g}^{*}$ which is graded-commutative, it has the structure of homotopy commutative and associative algebra, or $C_{\infty}$-algebra.

Throughout the text $\mathfrak{g}$ will be the free 2-nilpotent graded Lie algebra, with degree one generators in the finite dimensional vector space $V$ over a field $\mathbb{K}$ of characteristic 0 ,

$$
\mathfrak{g}=V \oplus \bigwedge^{2} V .
$$

The UEA $U\left(V \oplus \wedge^{2} V\right)$ arises naturally in physics in the universal Fock-like space of the parastatistics algebra introduced by H.S. Green [5](see also [3]). Here we will concentrate on the case when $V$ is an ordinary (even) vector space $V$, when the algebra $U \mathfrak{g}$ is the parafermionic algebra.

The aim of this note is to describe the Yoneda algebra $\mathscr{E}$ of the UEA $U \mathfrak{g}$, i.e., the cohomology $H^{\bullet}(\mathfrak{g}, \mathbb{K})$ with its $C_{\infty}$-structure induced by the isomorphism (1) through the homotopy transfer.

The cohomology space $H^{\bullet}(\mathfrak{g}, \mathbb{K})$ has a natural $G L(V)$-action. The decomposition of the $G L(V)$-module $H^{\bullet}(\mathfrak{g}, \mathbb{K})$ into irreducible Schur modules $V_{\lambda}$ is known since the work of Józefiak and Weyman [6]; it contains all $G L(V)$-modules with selfconjugated Young diagrams $\lambda=\lambda^{\prime}$ once and exactly once. The decomposition of $\mathscr{E}=H^{\bullet}(\mathfrak{g}, \mathbb{K})$ into Schur modules provides a powerful tool to handle its $C_{\infty}$-algebra structure.

\section{Artin-Schelter regularity}

Let $\mathfrak{g}$ be the 2-nilpotent graded Lie algebra $\mathfrak{g}=V \oplus \bigwedge^{2} V$ generated by the finite dimensional vector space $V$ having Lie bracket

$$
[x, y]:=\left\{\begin{array}{cl}
x \wedge y & x, y \in V \\
0 & \text { otherwise }
\end{array} .\right.
$$

We denote the Universal Enveloping Algebra $U \mathfrak{g}$ by $P S$ and will refer to it as parastatistics algebra (by some abuse ${ }^{1}$ ). The parastatistics algebra $P S(V)$ generated in $V$ is graded

$$
P S(V):=U \mathfrak{g}=U\left(V \oplus \bigwedge^{2} V\right)=T(V) /([[V, V], V]) .
$$

We shall write simply $P S$ when the space of generators $V$ is clear from the context.

Artin and Schelter [1] introduced a class of regular algebras sharing some "good" homological properties with the polynomial algebra $\mathbb{K}[V]$. These algebras were dubbed Artin-Schelter regular algebras (AS-regular algebra for short).

Definition 1. (AS-regular algebras) A connected graded algebra $\mathscr{A}=\mathbb{K} \oplus \mathscr{A}_{1} \oplus$ $\mathscr{A}_{2} \oplus \ldots$ is called Artin-Schelter regular of dimension $d$ if

(i) $\mathscr{A}$ has finite global dimension $d$,

(ii) $\mathscr{A}$ has finite Gelfand-Kirillov dimension,

\footnotetext{
${ }^{1}$ Strictly speaking $P S(V)$ is the creation parastatistics algebra, closed by creation operators alone.
} 
(iii) $\mathscr{A}$ is Gorenstein, i.e., $\operatorname{Ext}_{\mathscr{A}}^{i}(\mathbb{K}, \mathscr{A})=\delta^{i, d} \mathbb{K}$.

A general theorem claims that the UEA of a finite dimensional positively graded Lie algebra is an AS-regular algebra of global dimension equal to the dimension of the Lie algebra [4]. Hence the parastatistics algebra $P S$ is AS-regular of global dimension $d=\frac{\operatorname{dim} V(\operatorname{dim} V+1)}{2}$. In particular the finite global dimension of $P S$ implies that the ground field $\mathbb{K}$ has a minimal resolution $P_{\bullet}$ by projective left $P S$-modules $P_{n}$

$$
P_{\bullet}: \quad 0 \rightarrow P_{d} \rightarrow \cdots \rightarrow P_{n} \rightarrow \cdots \rightarrow P_{2} \rightarrow P_{1} \rightarrow P_{0} \stackrel{\varepsilon}{\rightarrow} \mathbb{K} \rightarrow 0 .
$$

Here $\mathbb{K}$ is a trivial left $P S$-module, the action being defined by the projection $\varepsilon$ onto $P S_{0}=\mathbb{K}$. Since $P S$ is positively graded and, in the category of positively graded modules over connected locally finite graded algebras, projective module is the same as free module [2], we have $P_{n} \cong P S \otimes E_{n}$ where $E_{n}$ are finite dimensional vector spaces.

The minimal projective resolution is unique (up to an isomorphism). Minimality implies that the complex $\mathbb{K} \otimes_{P S} P_{\bullet}$ has "zero differentials" hence

$$
H_{\bullet}\left(\mathbb{K} \otimes_{P S} P_{\bullet}\right)=\mathbb{K} \otimes_{P S} P_{\bullet}=E_{n} .
$$

One can calculate the derived functor $\operatorname{Tor}_{n}^{P S}(\mathbb{K}, \mathbb{K})$ using the resolution $P_{\bullet}$, it yields

$$
\operatorname{Tor}_{n}^{P S}(\mathbb{K}, \mathbb{K})=E_{n}
$$

The data of a minimal resolution of $\mathbb{K}$ by free $P S$-modules provides an easy way to find $\operatorname{Tor}_{n}^{P S}(\mathbb{K}, \mathbb{K})$. Conversely if the spaces $\operatorname{Tor}_{n}^{P S}(\mathbb{K}, \mathbb{K})$ are known, then one can constuct a minimal free resolution of $\mathbb{K}$.

The Gorenstein property guarantees that when applying the functor $\operatorname{Hom}_{P S}(-, P S)$ to the minimal free resolution $P_{\bullet}$ we get another minimal free resolution $P^{\bullet}:=$ $\operatorname{Hom}_{P S}\left(P_{\bullet}, P S\right)$ of $\mathbb{K}$ by right $P S$-modules

$$
P^{\bullet}: \quad 0 \leftarrow \mathbb{K} \leftarrow P_{d}^{\prime} \leftarrow \cdots \leftarrow P_{n}^{\prime} \leftarrow \cdots \leftarrow P_{2}^{\prime} \leftarrow P_{1}^{\prime} \leftarrow P_{0}^{\prime} \leftarrow 0
$$

with $P_{n}^{\prime} \cong E_{n}^{*} \otimes P S$. Note that by construction $E_{n}^{*}=\operatorname{Ext}_{P S}^{n}(\mathbb{K}, \mathbb{K})$, thus one has vector space isomorphisms [2]

$$
E_{n} \cong E_{n}^{*} \cong \operatorname{Tor}_{n}^{P S}(\mathbb{K}, \mathbb{K}) \cong \operatorname{Ext}_{P S}^{n}(\mathbb{K}, \mathbb{K}) .
$$

The Gorenstein property is an analog of the Poincaré duality, it implies $E_{d-n}^{*} \cong E_{n}$. The finite global dimension $d$ of $P S$ and the Gorenstein condition imply that its Yoneda algebra

$$
\mathscr{E}^{\bullet}:=\operatorname{Ext}_{P S}^{\bullet}(\mathbb{K}, \mathbb{K}) \cong \bigoplus_{n=0}^{d} E_{n}^{*}
$$

is Frobenius [10]. More on Gorenstein property you can find in the first autor's lecture "Poincaré duality for Koszul algebras" in the present volume. 


\section{Homology and cohomology of $\mathfrak{g}$}

A non-minimal projective(in fact free) resolution of $\mathbb{K}, C(\mathfrak{g}) \stackrel{\varepsilon}{\rightarrow} \mathbb{K}$ is given by the standard Chevalley-Eilenberg chain complex $C_{\bullet}(\mathfrak{g})=\left(U \mathfrak{g} \otimes_{\mathbb{K}} \wedge^{p} \mathfrak{g}, d_{p}\right)$ with differential maps

$$
\begin{aligned}
d_{p}(u & \left.\otimes x_{1} \wedge \ldots \wedge x_{p}\right)=\sum_{i}(-1)^{i+1} u x_{i} \otimes x_{1} \wedge \ldots \wedge \hat{x}_{i} \wedge \ldots \wedge x_{p} \\
& +\sum_{i<j}(-1)^{i+j} u \otimes\left[x_{i}, x_{j}\right] \wedge x_{1} \wedge \ldots \wedge \hat{x}_{i} \wedge \ldots \wedge \hat{x}_{j} \wedge \ldots \wedge x_{p}
\end{aligned}
$$

The resolution $C_{\bullet}(\mathfrak{g})$ calculates the homologies of the derived complex $\mathbb{K} \otimes_{P S} C_{\bullet}(\mathfrak{g})$

$$
E_{n}=\operatorname{Tor}_{n}^{P S}(\mathbb{K}, \mathbb{K}) \cong H_{n}\left(\mathbb{K} \otimes_{P S} C_{\bullet}(\mathfrak{g})\right)=H_{n}(\mathfrak{g}, \mathbb{K})
$$

coinciding with the homologies $H_{n}(\mathfrak{g}, \mathbb{K})$ of the Lie algebra $\mathfrak{g}$ with trivial coefficients. The derived complex $\mathbb{K} \otimes_{P S} C_{\bullet}(\mathfrak{g})$ is the chain complex with degrees $\bigwedge^{\bullet} \mathfrak{g}=\mathbb{K} \otimes_{P S} P S \otimes \bigwedge^{\bullet} \mathfrak{g}$ and differentials $\partial_{p}:=i d \otimes_{P S} d_{p}: \bigwedge^{p} \mathfrak{g} \rightarrow \bigwedge^{p-1} \mathfrak{g}$. One has

$$
\bigwedge^{p} \mathfrak{g}=\bigwedge^{p}\left(V \oplus \bigwedge^{2} V\right)=\bigoplus_{s+r=p} \bigwedge^{s}\left(\bigwedge^{2} V\right) \otimes \bigwedge^{r}(V)
$$

and differentials $\partial_{p=r+s}: \bigwedge^{s}\left(\bigwedge^{2} V\right) \otimes \bigwedge^{r}(V) \rightarrow \bigwedge^{s+1}\left(\bigwedge^{2} V\right) \otimes \bigwedge^{r-2}(V)$ are given by

$$
\begin{aligned}
\partial_{p}: & e_{i_{1} j_{1}} \wedge \ldots \wedge e_{i_{s} j_{s}} \otimes e_{1} \wedge \ldots \wedge e_{r} \mapsto \\
& \sum_{i<j}(-1)^{i+j} e_{i j} \wedge e_{i_{1} j_{1}} \wedge \ldots \wedge e_{i_{s} j_{s}} \otimes e_{1} \wedge \ldots \wedge \hat{e}_{i} \wedge \ldots \wedge \hat{e}_{j} \wedge \ldots \wedge e_{r} .
\end{aligned}
$$

The differential $\partial$ is induced by the Lie bracket $[\cdot, \cdot]: \bigwedge^{2} \mathfrak{g} \rightarrow \mathfrak{g}$, it identifies a pair of degree 1 generators $e_{i}, e_{j} \in$ with one degree 2 generator $e_{i j}:=\left(e_{i} \wedge e_{j}\right)=\left[e_{i}, e_{j}\right]$. The differential $\partial_{p}$ is the extension of $\partial_{2}:=-[\cdot, \cdot]$ as coderivation on $\wedge^{p} \mathfrak{g}$.

The dual cochain complex $\operatorname{Hom}_{P S}(C(\mathfrak{g}), \mathbb{K})=\left(\bigwedge^{\bullet} \mathfrak{g}^{*}, \delta\right)$ calculates cohomology ${ }^{2}$

$$
E_{n}^{*}=\operatorname{Ext}_{P S}^{n}(\mathbb{K}, \mathbb{K}) \cong H^{n}\left(\operatorname{Hom}_{P S}(C(\mathfrak{g}), \mathbb{K})\right)=H^{n}(\mathfrak{g}, \mathbb{K})
$$

The coboundary map $\delta^{p}: \bigwedge^{p} \mathfrak{g}^{*} \rightarrow \bigwedge^{p+1} \mathfrak{g}^{*}$ is transposed to the differential $\partial_{p+1}$

$$
\begin{gathered}
\delta^{p}: e_{i_{1} j_{1}}^{*} \wedge \ldots \wedge e_{i_{s} j_{s}}^{*} \otimes e_{l_{1}}^{*} \wedge \ldots \wedge e_{l_{r}}^{*} \mapsto \\
\sum_{k=1}^{s} \sum_{i_{k}<j_{k}}(-1)^{i+j} e_{i_{1} j_{1}}^{*} \wedge \ldots \wedge \hat{e}_{i_{k} j_{k}}^{*} \wedge \ldots \wedge e_{i_{s} j_{s}}^{*} \otimes e_{i_{k}}^{*} \wedge e_{j_{k}}^{*} \wedge e_{l_{1}}^{*} \wedge \ldots \wedge \ldots \wedge e_{l_{r}}^{*},
\end{gathered}
$$

it is (up to a conventional sign) the extension as derivation of the dualization of the Lie bracket $\delta^{1}:=[\cdot, \cdot]^{*}: \mathfrak{g}^{*} \rightarrow \bigwedge^{2} \mathfrak{g}^{*}$.Thus the algebra $\left(\bigwedge^{\bullet} \mathfrak{g}^{*}, \delta\right)$ equipped with $\delta$ is a (graded-)commutative DGA.

\footnotetext{
${ }^{2}$ In the presence of metric one has $\delta:=\partial^{*}$ (see below)
} 


\section{Homology of $\mathfrak{g}$ as a $G L(V)$-module}

An irreducible polynomial $G L(V)$-module $V_{\lambda}$ is called Schur module, it has a basis labelled by semistandard Young tableaux which are fillings of the Young diagram $\lambda$ with the numbers of the set $\{1, \ldots, \operatorname{dim} V\}$. The action of the linear group $G L(V)$ on the space $V$ of the generators of the Lie algebra $\mathfrak{g}$ induces a $G L(V)$-action on the UEA $P S=U \mathfrak{g} \cong S\left(V \oplus \Lambda^{2} V\right)$ and on the space $\wedge^{\bullet} \mathfrak{g} \cong \Lambda^{\bullet}\left(V \oplus \Lambda^{2} V\right)$.

In the presence of metric $g$ one has an identification $V \stackrel{g}{\cong} V^{*}$, and $\Lambda^{\bullet} \mathfrak{g} \stackrel{g}{\cong} \Lambda^{\bullet} \mathfrak{g}^{*}$. The adjoint operator $\partial_{p}^{*}: \bigwedge^{p} \mathfrak{g} \rightarrow \bigwedge^{p+1} \mathfrak{g}$ is defined by $g\left(\partial_{p}^{*} v, w\right)=g\left(v, \partial_{p+1} w\right)$. It turns out that the action of $\partial_{p}^{*}$ always takes the form (similar to the action of $\delta^{p}$ )

$$
\begin{gathered}
\partial_{p}^{*}: e_{i_{1} j_{1}} \wedge \ldots \wedge e_{i_{s} j_{s}} \otimes e_{l_{1}} \wedge \ldots \wedge e_{l_{r}} \mapsto \\
\sum_{k=1}^{s} \sum_{i_{k}<j_{k}}(-1)^{i+j} e_{i_{1} j_{1}} \wedge \ldots \wedge \hat{e}_{i_{k} j_{k}} \wedge \ldots \wedge e_{i_{s} j_{s}} \otimes e_{i_{k}} \wedge e_{j_{k}} \wedge e_{l_{1}} \wedge \ldots \wedge \ldots \wedge e_{l_{r}},
\end{gathered}
$$

It is obvious that the maps $\partial$ and $\partial^{*}$ both commute with the $G L(V)$-action. The Laplacian $\Delta=\oplus_{p \geq 0} \Delta_{p}$ of the pair $(\mathfrak{g}, g)$ is defined to be the self-adjoint operator

$$
\Delta_{p}=\partial_{p+1} \partial_{p+1}^{*}+\partial_{p}^{*} \partial_{p} \in \operatorname{End}\left(\bigwedge^{\mathrm{p}} \mathfrak{g}\right)
$$

Its kernel is a complete set of representatives for the homology classes in $H_{p}(\mathfrak{g}, \mathbb{K})$

$$
\operatorname{ker} \Delta_{p} \cong H_{p}(\mathfrak{g}, \mathbb{K})
$$

The decomposition of the $G L(V)$-module $H_{n}(\mathfrak{g}, \mathbb{K})$ into irreducible polynomial representations $V_{\lambda}$ is given by the following theorem;

Theorem 1 (Józefiak and Weyman [6], Sigg [11]). The homology $H_{\bullet}(\mathfrak{g}, \mathbb{K})$ of the 2-nilpotent Lie algebra $\mathfrak{g}=V \oplus \bigwedge^{2} V$ decomposes into irreducible $G L(V)$-modules

$$
H_{n}(\mathfrak{g}, \mathbb{K})=H_{n}\left(\bigwedge^{\bullet} \mathfrak{g}, \partial\right) \cong \operatorname{Tor}_{n}^{P S}(\mathbb{K}, \mathbb{K})(V) \cong \bigoplus_{\lambda: \lambda=\lambda^{\prime}} V_{\lambda}
$$

where the sum is over self-conjugate Young diagrams $\lambda$ such that $n=\frac{1}{2}(|\lambda|+r(\lambda))$. The data $H_{n}(\mathfrak{g}, \mathbb{K})=\operatorname{Tor}_{n}^{P S}(\mathbb{K}, \mathbb{K})$ encodes the minimal free resolution $P_{\bullet}$ (cf. (3)).

The Euler characteristics of $P_{\bullet}$ implies an identity about the $G L(V)$-characters

$$
\operatorname{chPS}(V) \cdot \operatorname{ch}\left(\bigoplus_{\lambda: \lambda=\lambda^{\prime}}(-1)^{\frac{1}{2}(|\lambda|+r(\lambda))} V_{\lambda}\right)=1 .
$$

The character of a Schur module $V_{\lambda}$ is the Schur function, $\operatorname{ch} V_{\lambda}=s_{\lambda}(x)$. Due to the Poincaré-Birkhoff-Witt theorem $\operatorname{ch} P S(V)=\operatorname{ch} S\left(V \oplus \wedge^{2} V\right)$ thus the identity reads

$$
\prod_{i} \frac{1}{\left(1-x_{i}\right)} \prod_{i<j} \frac{1}{\left(1-x_{i} x_{j}\right)} \sum_{\lambda: \lambda=\lambda^{\prime}}(-1)^{\frac{1}{2}(|\lambda|+r(\lambda))} s_{\lambda}(x)=1 .
$$


But the latter identity is nothing but rewriting of the Littlewood identity [6]. The moral is that the Littlewood identity reflects a homological property of the algebra $P S$, namely the above particular structure of the minimal projective (free) resolution of $\mathbb{K}$ by $P S$-modules.

\section{Homotopy algebras $A_{\infty}$ and $C_{\infty}$}

Definition 2. ( $A_{\infty}$-algebra) A homotopy associative algebra, or $A_{\infty}$-algebra, over $\mathbb{K}$ is a $\mathbb{Z}$-graded vector space $A=\bigoplus_{i \in \mathbb{Z}} A^{i}$ endowed with a family of graded mappings (operations)

$$
m_{n}: A^{\otimes n} \rightarrow A, \quad \operatorname{deg}\left(m_{n}\right)=2-n \quad n \geq 1
$$

satisfying the Stasheff identities $\mathbf{S I}(\mathbf{n})$ for $n \geq 1$

$$
\sum_{r+s+t=n}(-1)^{r+s t} m_{r+1+t}\left(I d^{\otimes r} \otimes m_{s} \otimes I d^{\otimes t}\right)=0 \quad \mathbf{S I}(\mathbf{n})
$$

where the sum runs over all decompositions $n=r+s+t$.

Here we assume the Koszul sign convention $(f \otimes g)(x \otimes y)=(-1)^{|g||x|} f(x) \otimes g(y)$. We define the shuffle product $S h_{p, q}: A^{\otimes p} \otimes A^{\otimes q} \rightarrow A^{\otimes p+q}$ throughout the expression

$$
\left(a_{1} \otimes \ldots \otimes a_{p}\right) \amalg\left(a_{p+1} \otimes \ldots \otimes a_{p+q}\right)=\sum_{\sigma \in S h_{p, q}} \operatorname{sgn}(\sigma) a_{\sigma^{-1}(1)} \otimes \ldots \otimes a_{\sigma^{-1}(p+q)}
$$

where the sum runs over all $(p, q)$-shuffles $S h_{p, q}$, i.e., over all permutations $\sigma \in S_{p+q}$ such that $\sigma(1)<\sigma(2)<\ldots<\sigma(p)$ and $\sigma(p+1)<\sigma(p+2)<\ldots<\sigma(p+q)$.

Definition 3. $\left(C_{\infty}\right.$-algebra [7]) A homotopy commutative algebra, or $C_{\infty}$-algebra, is an $A_{\infty}$-algebra $\left\{A, m_{n}\right\}$ such that each operation $m_{n}$ vanishes on non-trivial shuffles

$$
m_{n}\left(\left(a_{1} \otimes \ldots \otimes a_{p}\right) \amalg\left(a_{p+1} \otimes \ldots \otimes a_{n}\right)\right)=0, \quad 1 \leq p \leq n-1 .
$$

In particular for $m_{2}$ we have $m_{2}(a \otimes b \pm b \otimes a)=0$, so a $C_{\infty}$-algebra such that $m_{n}=0$ for $n \geq 3$ is a (super-)commutative DGA.

A morphism of two $A_{\infty}$-algebras $A$ and $B$ is a family of graded maps $f_{n}: A^{\otimes n} \rightarrow B$ for $n \geq 1$ with $\operatorname{deg} f_{n}=1-n$ such that the following conditions hold

$$
\sum_{r+s+t=n}(-1)^{r+s t} f_{r+1+t}\left(I d^{\otimes r} \otimes m_{s} \otimes I d^{\otimes r}\right)=\sum_{1 \leq r \leq n}(-1)^{S} m_{r}\left(f_{i_{1}} \otimes f_{i_{2}} \otimes \ldots \otimes f_{i_{r}}\right)
$$

where the sum is on all decompositions $i_{1}+\ldots+i_{r}=n$ and the sign on RHS is determined by $S=\sum_{k=1}^{r-1}(r-k)\left(i_{k}-1\right)$. The morphism $f$ is a quasi-isomorphism of $A_{\infty}$-algebras if $f_{1}$ is a quasi-isomorphism. It is strict if $f_{i}=0$ for all $i \neq 1$. The identity morphism of $A$ is the strict morphism $f$ such that $f_{1}$ is the identity of $A$.

A morphism of $C_{\infty}$-algebras is a morphism of $A_{\infty}$-algebras vanishing on nontrivial shuffles $f_{n}\left(\left(a_{1} \otimes \ldots \otimes a_{p}\right) \amalg\left(a_{p+1} \otimes \ldots \otimes a_{n}\right)\right)=0,1 \leq p \leq n-1$. 


\section{Homotopy Transfer Theorem}

Lemma 1. Every cochain complex $(A, d)$ of vector spaces over a field $\mathbb{K}$ has its cohomology $H^{\bullet}(A)$ as a deformation retract.

One can always choose a vector space decomposition of the cochain complex $(A, d)$ such that $A^{n} \cong B^{n} \oplus H^{n} \oplus B^{n+1}$ where $H^{n}$ is the cohomology and $B^{n}$ is the space of coboundaries, $B^{n}=d A^{n-1}$. We choose a homotopy $h: A^{n} \rightarrow A^{n-1}$ which identifies $B^{n}$ with its copy in $A^{n-1}$ and is 0 on $H^{n} \oplus B^{n+1}$. The projection $p$ to the cohomology and the cocycle-choosing inclusion $i$ given by $A^{n} \underset{i}{\stackrel{p}{<}} H^{n}$ are chain homomorphisms (satisfying the additional conditions $h h=0, h i=0$ and $p h=0$ ). With these choices done the complex $\left(H^{\bullet}(A), 0\right)$ is a deformation retract of $(A, d)$

$$
h \bigcirc(A, d) \underset{i}{\stackrel{p}{\rightleftarrows}}\left(H^{\bullet}(A), 0\right), \quad p i=I d_{H^{\bullet}(A)}, \quad i p-I d_{A}=d h+h d .
$$

Let now $(A, d, \mu)$ be a DGA, i.e., $A$ is endowed with an associative product $\mu$ compatible with $d$. The cochain complexes $(A, d)$ and its contraction $H^{\bullet}(A)$ are homotopy equivalent, but the associative structure is not stable under homotopy equivalence. However the associative structure on $A$ can be transferred to an $A_{\infty}$-structure on a homotopy equivalent complex, a particular interesting complex being the deformation retract $H^{\bullet}(A)$. For a friendly introduction to homotopy transfer theorems in much boarder context we send the reader to the textbook [9], see chapter 9.

Theorem 2 (Kadeishvili [7]). Let $(A, d, \mu)$ be a (commutative) DGA over a field $\mathbb{K}$. There exists a $A_{\infty}$-algebra $\left(C_{\infty}\right.$-algebra) structure on the cohomology $H^{\bullet}(A)$ and a $A_{\infty}\left(C_{\infty}\right)$-quasi-isomorphism $f_{i}:\left(\otimes^{i} H^{\bullet}(A),\left\{m_{i}\right\}\right) \rightarrow(A,\{d, \mu, 0,0, \ldots\})$ such that the inclusion $f_{1}=i: H^{\bullet}(A) \rightarrow A$ is a cocycle-choosing homomorphism of cochain complexes. The differential $m_{1}$ on $H^{\bullet}(A)$ is zero $\left(m_{1}=0\right)$ and $m_{2}$ is strictly associative operation induced by the multiplication on A. The resulting structure is unique up to quasi-isomorphism.

Kontsevich and Soibelman [12] gave an explicit expressions for the higher operations of the induced $A_{\infty}$-structure as sums over decorated planar binary trees with one root where all leaves are decorated by the inclusion $i$, the root by the projection $p$ the vertices by the product $\mu$ of the (commutative) DGA $(A, d, \mu)$ and the internal edges by the homotopy $h$. The $C_{\infty}$-structure implies additional symmetries on trees. We will make use of the graphic representation for the binary operation on $H^{\bullet}(A)$

$$
m_{2}(x, y):=p \mu(i(x), i(y)) \quad \text { or } \quad m_{2}=
$$

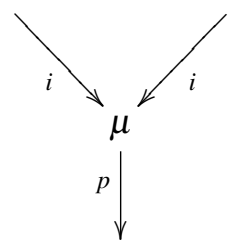


and the ternary one $m_{3}(x, y, z)=p \mu(i(x), h \mu(i(y), i(z)))-p \mu(h \mu(i(x), i(y)), i(z))$ being the sum of two planar binary trees with three leaves
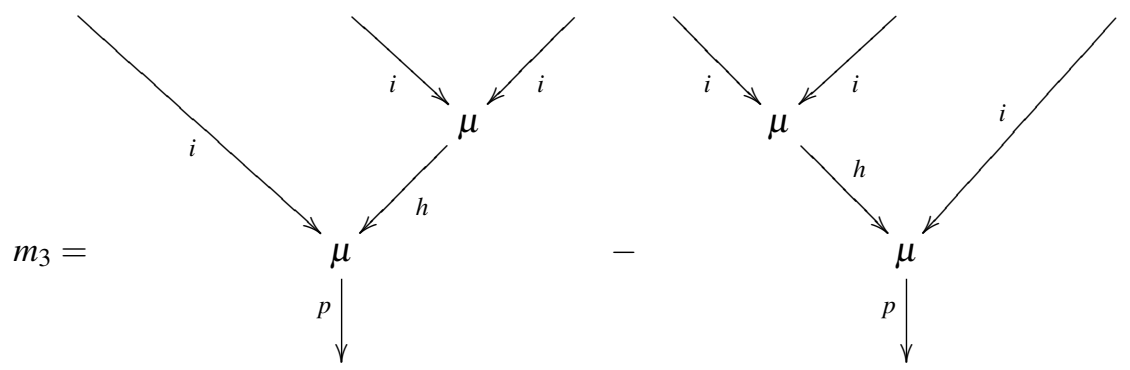

Theorem 3. The cohomology $H^{\bullet}(\mathfrak{g}, \mathbb{K}) \cong \operatorname{Ext}_{P S}^{\bullet}(\mathbb{K}, \mathbb{K})$ of the 2-nilpotent graded Lie algebra $\mathfrak{g}=V \otimes \bigwedge^{2} V$ is a homotopy commutative algebra which is generated in degree 1 (i.e., in $H^{1}(\mathfrak{g}, \mathbb{K})$ ) by the operations $m_{2}$ and $m_{3}$.

Sketch of the proof. Let us choose a metric $g(\cdot, \cdot)=\langle\cdot, \cdot\rangle$ on the vector space $V$ and an orthonormal basis $\left\langle e_{i}, e_{j}\right\rangle=\delta_{i j}$. The choice induces a metric on $\wedge^{\bullet} \mathfrak{g} \stackrel{g}{\cong} \Lambda^{\bullet} \mathfrak{g}^{*}$.

Due to the isomorphisms $\operatorname{Tor}_{n}^{P S}(\mathbb{K}, \mathbb{K}) \cong \operatorname{Ext}_{P S}^{n}(\mathbb{K}, \mathbb{K})$ (see eq. 6) and $V \cong V^{*}$ the theorem 1 implies the decomposition of $H^{\bullet}(\mathfrak{g}, \mathbb{K})$ into irreducible $G L(V)$-modules

$$
H^{n}(\mathfrak{g}, \mathbb{K}) \cong H^{n}\left(\bigwedge \mathfrak{g}^{*}, \delta\right) \cong \operatorname{Ext}_{P S}^{n}(\mathbb{K}, \mathbb{K})\left(V^{*}\right) \cong \underset{\lambda: \lambda=\lambda^{\prime}}{\bigoplus} V_{\lambda}
$$

where the sum is over self-conjugate diagrams $\lambda$ such that $n=\frac{1}{2}(|\lambda|+r(\lambda))$.

In the presence of metric $g$ the differential $\delta$ is identified with the adjoint of $\partial$, $\delta: \stackrel{g}{=} \partial^{*}$ while $\partial$ plays the role of a homotopy. In view of lemma 1 we have the cohomology $H^{\bullet}\left(\bigwedge^{\bullet} \mathfrak{g}^{*}, \delta^{\bullet}\right)$ as deformation retract of the complex $\left(\bigwedge^{\bullet} \mathfrak{g}^{*}, \delta^{\bullet}\right)$,

$$
p i=I d_{H^{\bullet}\left(\wedge^{\bullet} \mathfrak{g}^{*}\right)}, \quad i p-I d_{\wedge^{\bullet} \mathfrak{g}^{*}}=\delta \delta^{*}+\delta^{*} \delta, \quad \delta^{*} \stackrel{g}{=} \partial .
$$

Here the projection $p$ identifies the subspace $\operatorname{ker} \delta \cap \operatorname{ker} \delta^{*}$ with $H^{\bullet}\left(\bigwedge^{\bullet} \mathfrak{g}^{*}\right)$, which is the orthogonal complement of the space of the coboundaries im $\delta$. The cocyclechoosing homomorphism $i$ is $I d$ on $H^{\bullet}\left(\bigwedge^{\bullet} \mathfrak{g}^{*}\right)$ and zero on coboundaries.

We apply the Kadeishvili homotopy transfer Theorem 2 for the commutative DGA $\left(\wedge^{\bullet} \mathfrak{g}^{*}, \mu, \delta^{\bullet}\right)$ and its deformation retract $H^{\bullet}\left(\bigwedge^{\bullet} \mathfrak{g}^{*}\right) \cong H^{\bullet}(\mathfrak{g}, \mathbb{K})$ and conclude that the cohomology $H^{\bullet}(\mathfrak{g}, \mathbb{K})$ is a $C_{\infty}$-algebra.

The Kontsevich and Soibelman tree representations of the operations $m_{n}$ provide explicit expressions. Let us take $\mu$ to be the super-commutative product $\wedge$ on the DGA $\left(\bigwedge^{\bullet} \mathfrak{g}^{*}, \delta^{\bullet}\right)$. The projection $p$ maps onto the Schur modules $V_{\lambda}$ with $\lambda=\lambda^{\prime}$.

The binary operation on the degree 1 generators $e_{i} \in H^{1}(\mathfrak{g}, \mathbb{K})$ is trivial, one gets

$$
m_{2}\left(e_{i}, e_{j}\right)=p\left(e_{i} \wedge e_{j}\right)=0 \quad p\left(V_{\left(1^{2}\right)}\right)=0 .
$$

Hence $H^{\bullet}(\mathfrak{g}, \mathbb{K})$ could not be generated in $H^{1}(\mathfrak{g}, \mathbb{K})$ as algebra with product $m_{2}$. 
The ternary operation $m_{3}$ restricted to $H^{1}(\mathfrak{g}, \mathbb{K})$ is nontrivial, indeed one has

$$
\begin{aligned}
m_{3}\left(e_{i}, e_{j}, e_{k}\right) & =p\left\{e_{i} \wedge \partial\left(e_{j} \wedge e_{k}\right)-\partial\left(e_{i} \wedge e_{j}\right) \wedge e_{k}\right\}=p\left\{e_{i j} \wedge e_{k}-e_{i} \wedge e_{j k}\right\} \\
& =p\left\{\left(e_{i j} \wedge e_{k}+e_{j k} \wedge e_{i}+e_{k i} \wedge e_{j}\right)-e_{k i} \wedge e_{j}\right\}=e_{i k} \wedge e_{j} \in H^{2}(\mathfrak{g}, \mathbb{K})
\end{aligned}
$$

The completely antisymmetric combination in the brackets (...) spans the Schur module $V_{\left(1^{3}\right)}, p\left(e_{i j} \wedge e_{k}+e_{j k} \wedge e_{i}+e_{k i} \wedge e_{j}\right)=0$ yields a Jacobi-type identity. The monomials $e_{i j} \wedge e_{k}$ modulo $V_{\left(1^{3}\right)}$ span a Schur module $V_{(2,1)} \in H^{2}(\mathfrak{g}, \mathbb{K})$ with basis in bijection with the semistandard Young tableaux $e_{i k} \wedge e_{j} \leftrightarrow$\begin{tabular}{ll}
$i$ & $j$ \\
$k$ & and $e_{i j} \wedge e_{k} \leftrightarrow, i$ \\
\hline$j$ & $k$
\end{tabular}

We check the symmetry condition on ternary operation $m_{3}$ in $C_{\infty}$-algebra; indeed $m_{3}$ vanishes on the (signed) shuffles $S h_{1,2}$ and $S h_{2,1}$

$m_{3}\left(e_{i} \uplus e_{j} \otimes e_{k}\right)=m_{3}\left(e_{i}, e_{j}, e_{k}\right)-m_{3}\left(e_{j}, e_{i}, e_{k}\right)+m_{3}\left(e_{j}, e_{k}, e_{i}\right)=0=m_{3}\left(e_{i} \otimes e_{j} \uplus e_{k}\right)$.

It is important that in the complexes $\left(\bigwedge^{p} \mathfrak{g}, \partial_{p}\right)$ and $\left(\bigwedge^{p} \mathfrak{g}^{*}, \delta^{p}\right)$ two different degees are involved; one is the homological degree $p:=r+s$ counting the number of $\mathfrak{g}$-generators, while the second is the tensor degree $t:=2 s+r$ (also called weight). The differentials $\partial$ and $\delta$ preserve the tensor degree $t$ but the spaces $H_{n}(\mathfrak{g}, \mathbb{K})$ and $H^{n}(\mathfrak{g}, \mathbb{K})$ are not homogeneous in $t$. The operation $m_{n}$ is bigraded by homological and tensor gradings of bidegree $(p, t)=(2-n, 0)$. The bi-grading impose the vanishing of many higher products.

On the level of Schur modules the ternary operation glues three fundamental $G L(V)$-representations $V_{\square}$ into a Schur module $V_{(2,1)}$. By iteration of the process of gluing boxes we generate all elementary hooks $V_{k}:=V_{\left(k+1,1^{k}\right)}$,

$m_{3}\left(V_{\square}, V_{\square}, V_{\square}\right)=V_{\square}, \quad m_{3}\left(V_{\square}, V_{\square}, V_{\square}\right)=V_{\square}, \ldots, m_{3}\left(V_{k}, V_{0}, V_{0}\right)=V_{k+1}$.

In our context the more convenient notation for Young diagrams is due to Frobenius: $\lambda:=\left(a_{1}, \ldots, a_{r} \mid b_{1}, \ldots b_{r}\right)$ stands for a diagram $\lambda$ with $a_{i}$ boxes in the $i$-th row on the right of the diagonal, and with $b_{i}$ boxes in the $i$-th column below the diagonal and the rank $r=r(\lambda)$ is the number of boxes on the diagonal.

For self-dual diagrams $\lambda=\lambda^{\prime}$, i.e., $a_{i}=b_{i}$ we set $V_{a_{1}, \ldots, a_{r}}:=V_{\left(a_{1}, \ldots, a_{r} \mid a_{1}, \ldots a_{r}\right)}$ when $a_{1}>a_{2}>\ldots>a_{r} \geq 0$ (and set the convention $V_{a_{1}, \ldots, a_{r}}:=0$ otherwise). Any two elementary hooks $V_{a_{1}}$ and $V_{a_{2}}$ can be glued together by the binary operation $m_{2}$, the decomposition of $m_{2}\left(V_{a_{1}}, V_{a_{2}}\right) \cong m_{2}\left(V_{a_{2}}, V_{a_{1}}\right)$ is given by

$$
m_{2}\left(V_{a_{1}}, V_{a_{2}}\right)=V_{a_{1}, a_{2}} \oplus\left(\bigoplus_{i=1}^{a_{2}} V_{a_{1}+i, a_{2}-i}\right) \quad a_{1} \geq a_{2}
$$

where the "leading" term $V_{a_{1}, a_{2}}$ has the diagram with minimal height. Hence any $m_{2}$-bracketing of the hooks $V_{a_{1}}, V_{a_{2}} \ldots, V_{a_{r}}$ yields ${ }^{3}$ a sum of $G L(V)$-modules

\footnotetext{
${ }^{3}$ The operation $m_{2}$ is associative thus the result does not depend on the choice of the bracketing.
} 


$$
m_{2}\left(\ldots m_{2}\left(m_{2}\left(V_{a_{1}}, V_{a_{2}}\right), V_{a_{3}}\right), \ldots, V_{a_{r}}\right)=V_{a_{1}, \ldots, a_{r}} \oplus \ldots
$$

whose module with minimal height is precisely $V_{a_{1}, \ldots, a_{r}}$. We conclude that all elements in the $C_{\infty}$-algebra $H^{\bullet}(\mathfrak{g}, \mathbb{K})$ can be generated in $H^{1}(\mathfrak{g}, \mathbb{K})$ by $m_{2}$ and $m_{3}$.

Acknowledgements We are grateful to Jean-Louis Loday for many enlightening discussions and his encouraging interest. The work was supported by the French-Bulgarian Project Rila under the contract Egide-Rila N112.

\section{References}

1. M. Artin, W.F. Schelter, Graded algebras of global dimension 3. Adv. Math. 66(1987), 171216.

2. H. Cartan. Homologie et cohomologie d' une algèbre graduée. Séminaire Henri Cartan, 11(1958), 1-20.

3. M. Dubois-Violette, T. Popov, Homogeneous Algebras, Statistics and Combinatorics, LMP 61(2002), 159-170.

4. G. Floystad, J.E. Vayne, Artin-Schelter regular algebras of dimension five, Algebra, Geometry, and Mathematical Physics, Banach Center Publications, 93, (2011), 19-39.

5. H. S. Green, A Generalized Method of Field Quantization, Phys. Rev. 90(1953), 270-273.

6. T. Józefiak, J. Weyman, Representation-theoretic interpretation of a formula of D. E. Littlewood, Math. Proc. Cambridge Phil. Soc., 103(1988), 193-196.

7. T. Kadeishvili, The $A_{\infty}$-algebra Structure and Cohomology of Hochschild and Harrison, Proc. of Tbil. Math. Inst. 91(1988), 19-27.

8. J.-L. Loday and T. Popov, Parastatistics Algebra, Young Tableaux and the Super Plactic Monoid. Int. J. Geom. Meth. in Mod. Phys. 5 (2008), 1295-1314.

9. J.-L. Loday, B. Vallette, Algebraic Operads, Grundlehren Math. Wiss. 346, Springer, Heidelberg, 2012.

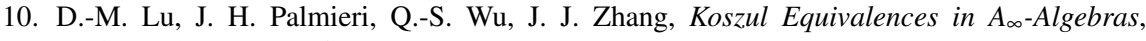
arXiv[math.RA]0710.5492.

11. S. Sigg, Laplacian and homology of free 2-step nilpotent Lie algebras, J. Algebra 185(1996), 144-161.

12. M. Kontsevich, Y. Soibelman, Deformations of algebras over operads and the Deligne conjecture, Math. Phys. Stud., 21(2000), Kluwer Acad. Publ., Dordrecht, 255-307. 